\title{
Novel Directions in Adjuvant Chemotherapy for Early Stage Epithelial Ovarian Cancer
}

\author{
Derya Kilic Sakarya ${ }^{1 *}$, M Hakan Yetimalar², Demir Ozbasar ${ }^{3}$
}

\begin{abstract}
Treatment of early stage ovarian cancer remains controversial despite advances in chemotherapeutic options. Over the past 30 years, molecular and clinicopathologic studies accelerated and treatment of ovarian cancer has undoubtedly improved although there is a debate as to whether this impacts outcome or not. More recently, the introduction of targeted therapy started a new era. Probably it is because early stage disease comprises a small portion of the epithelial ovarian cancer, studies have mostly ignored this group and still there is no clear consensus regarding systemic treatment of early-stage lesions. However this group of patients has the best chance of cure. In this review, we focus on current developments in the treatment of early stage ovarian cancer and query the options.
\end{abstract}

Keywords: Adjuvant chemotherapy - cytoreductive surgery - early stage ovarian cancer - treatment

Asian Pac J Cancer Prev, 16 (10), 4157-4160

\section{Introduction}

Only $25 \%$ of patients with epithelial ovarian cancer (EOC) are diagnosed at early stage when disease limited to pelvis (Siegel et al., 2013). Although prognosis of early stage disease (5-year survival rate is $80-90 \%$ ) is markedly better than advanced stage disease (5-year survival rate is $<30 \%$ ), some reports suggest a considerable recurrence rate of 15-40\% (Chan et al., 2010; Collinson et al., 2014, Tognon et al., 2013; Arikan et al., 2014). It is a known fact that surgical staging is critically important in early stage ovarian cancer management as in late stage. An accurate and complete staging, especially with leaving no residual tumoral tissue after surgery increases patient's chance to benefit from treatment and plays a key role in determining the chemotherapy regimen and duration of adjuvant chemotherapy. Due to low incidence and good prognosis of early stage ovarian cancer, early studies of adjuvant treatment are limited and provide unsatisfactory data about survival advantage. Subsequent researches focused on determining major prognostic factors, increasing success rate of surgical staging and developing adjuvant and neo-adjuvant therapy procedures, which may increase survival rates. Since stage 1a-1b grade 1 tumors have an excellent prognosis with a survival rate of around $90 \%$, some authors suggested that chemotherapy may not be necessary for this group (Chan et al., 2010). Adjuvant chemotherapy is recommended for all other early stage ovarian tumors.
In addition, for patients with early stage disease high risk criteria were defined to identify the patients with high risk for relaps and, who are candidates for adjuvant chemotherapy (Chan et al., 2010). These are; stage 1c-2 disease, clear cell histology (any stage) and grade 3 tumors. Women with these criteria have a 5-year survival rate of $40-80 \%$ and it is at least $90 \%$ for tumors limited to ovaries (stage 1a-1b) and well-differentiated (grade 1) (Collinson et al., 2014; Tognon et al., 2013; Chan et al., 2010). Whether intermediate differentiated (grade 2) tumors are in high risk group and adjuvant treatment is necessary after optimal surgery in early stage remains a matter of dispute (Lazarov et al., 2013; Malpica et al., 2004).

Two prospective European studies and their combined analysis provided important data on adjuvant chemotherapy. The International Collaberative Ovarian Neoplasm (ICON-1) and Adjuvant Chemotherapy in Ovarian Neoplasms (ACTION) studies involved 925 patients from 13 European countries with 10 years of observation (Trimbos et al., 2003; Colombo et al., 2003). Similar randomization procedures were performed and benefit of adjuvant chemotherapy in early stage ovarian cancer was investigated. European Gynecologic Oncology Group published the combined analysis of ICON-1 and ACTION studies. According to this analysis, 477 patients with early stage ovarian cancer from ICON-1 study (stage 1 grade 1-2-3 cases) and 448 patients with early stage ovarian cancer from ACTION study (stage 
1a-1b grade $2-3$, stage 1c grade 1-2-3, stage 2 a, clear cell stage 1-2) were randomized into two groups: first group had platin based adjuvant chemotherapy after surgery and second group was only followed-up without chemotherapy after surgery. Increased overall survival (OS) $(82 \%$ in adjuvant chemotherapy group and $74 \%$ in follow-up group) and progression free survival (PS) (76\% in adjuvant chemotherapy group and $65 \%$ in follow-up group) rates were observed after a follow-up period of longer than 4 years. Although not all patients had complete surgical staging, subgroup analyses of these patients were published (Trimbos et al., 2003; Trimbos et al., 2010). According to European Organization for Research and Treatment of Cancer (EORTC)-ACTION joint analysis:

i) In 297 patients without complete staging, PS and OS rates were significantly higher for adjuvant chemotherapy group when compared to follow-up group.

ii) In 224 patients of follow-up group, complete surgical staging was related to markedly increased PS and OS rates when compared to incomplete surgical staging group.

Limitations of these studies were application of different chemotherapy regimens and lack of surgical staging criteria.

In addition, a meta-analysis of 5 randomized studies on early stage ovarian cancer included 1277 patients and role of adjuvant chemotherapy in early stage disease was investigated (Winter-Roach et al., 2012). Similar to previous study, this meta-analysis also showed increased PS and OS rates with adjuvant chemotherapy.

i) Further analysis of patients without residual disease showed similar OS rates of adjuvant chemotherapy group and follow-up group. In patients with residual disease, survival rate was higher in adjuvant chemotherapy group when compared to follow-up group.

ii) In early stage patients meeting high risk criteria, survival rates were higher for adjuvant chemotherapy group when compared to follow-up group. In the group of patients with low risk, no survival benefit of adjuvant chemotherapy was observed.

All of these studies support the use of adjuvant chemotherapy in high risk patients with early stage ovarian cancer and patients with incomplete staging, while the use of chemotherapy in low risk patients with complete staging was considered as unnecessary. Further prospective clinical trials are needed to determine the necessity of chemotherapy in patients with one or more high risk criteria who undergone optimal surgery.

In this context, treatment algorithms published by National Comprehensive Cancer Network (NCCN) recommend follow-up rather than adjuvant chemotherapy for stage $1 \mathrm{a}-1 \mathrm{~b}$, grade 1 patients with complete surgical staging and use of adjuvant chemotherapy for patients with high risk disease (stage $1 \mathrm{c}-2$, grade 3 orany stage clear cell cancer) (recurrence rate of this group is about 50\%) (National Comprehensive Cancer Network (NCCN)., 2014). While adjuvant chemotherapy is usually recommended for grade 2 patients, observation is another alternative for these patients in the absence of other risk factors (Malpica et al., 2004; Lazarov et al., 2013).

\section{Optimal Intravenous Chemotherapy Regimen and Duration of Treatment}

Currently, adjuvant chemotherapy is recommended for patients with high risk criteria and early stage patients with incomplete staging. Although optimal regimen is undetermined, most of the clinicians apply platin based combined treatment (paclitaxel+carboplatin) because of its proven efficiency in advanced stage ovarian cancer patients (NCCN., 2014; Adams et al., 2010). According to the limited data on optimal regimen, however, single agent carboplatin can be an alternative for treatment (Adams et al., 2010). Well designed prospective studies are needed to determine the optimal chemotherapy regimen for these patients.

Optimal treatment duration for EOC is unknown. Considering patient's risk factors and tolerance to treatment, treatment should be individualized as at least 3 cycles.

Gynecologic Oncology Group (GOG) published GOG157 study which compared the effects of randomized 3 and 6 cycles of paclitaxel $\left(175 \mathrm{mg} / \mathrm{m}^{2} 3\right.$ hours iv infusion) and carboplatin (AUC 7.5; following paclitaxel) combination theraphy on survival in early stage patients with high risk criteria (Bell et al., 2006). 457 patients were involved in 3 years and patients were evaluated after 6.8 years of followup. More toxicity (neurotoxicity, granulocytopenia and anemia) was observed in 6 cycles chemotherapy group, while there were no statistically significant differences between groups in terms of recurrence and 5 year survival rates. In the subgroup analysis of the same study, patients with serous tumors were analyzed and significantly lower recurrence rates were observed in the group of 6 cycles of treatment when compared to the group of 3 cycles of treatment. There were no significant differences in other histological types (Chan et al., 2010).

GOG 175 study investigated whether 3 cycles carboplatin and paclitaxel combination followed by 24 weeks paclitaxel $\left(60 \mathrm{mg} / \mathrm{m}^{2}\right)$ treatment has positive effects on the outcomes. Although recurrence rate was $20 \%$ lower in extended paclitaxel group, this was statistically unsignificant and OS ratios were also similar (Mannel et al., 2011).

\section{Should Maintenance Treatment be Considered for Adjuvant Treatment of Early Stages?}

Present studies show that in early stage ovarian cancer, maintenance treatment is not related to decrease in recurrence rate or increase in OS, but increases the rate of toxicity (Mannel et al., 2011). The International Collaberative Ovarian Neoplasm-7 (ICON-7) trial did enroll a small group of women with high risk stage 1 epithelial ovarian cancer but there was no evident benefit of bevacizumab obtained. But it should be considered that the subgroup was small as it was just $9 \%$ of 1.528 patients (Perren et al., 2011). Arbeitsgemeinschaft Gynaekologische Onkologie Studiengruppe Ovarialkarzinom Group-17 (BOOST/ AGO-OVAR 17) is an undergoing study comparing 15 versus 30 month bevacizumab in the 
maintenance setting and also enrolls stage $1 \mathrm{C}$ disease (NCT01462890). There are no randomized trials evaluating biological agents in high risk early disease, and the only agent thus for evaluated has been bevacizumab (Perren et al., 2011). Further trials are warrented.

\section{Is Intraperitoneal Chemotherapy an Option of Adjuvant Therapy?}

Adjuvant intraperitoneal chemotherapy in early stage ovarian cancer is still at the experimental stage and being investigated by clinical studies (Raja et al., 2012).

Use of intraperitoneal P32 in early stage ovarian cancer was also studied. It was concluded that intraperitoneal P32 theraphy is not effective but increases toxicity (especially bone toxicity). At the present time, total abdominal radiation and intraperitoneal isotopes are old treatment modalities, instead adjuvant chemotherapy is the preferred treatment (Young et al., 2003).

\section{Adjuvant treatment after fertility sparing surgery}

Unilateral salpingo-oophorectomy in stage 1a G1/G2 young women who wish to preserve their childbearing potential is related to minimally increased recurrence risk (Satoh et al., 2010; Karimi et al., 2011). In these patients, a careful surgical staging must be performed considering grade and tumor characteristics. Stage 1c disease is less clear and careful consideration of subgroups of stage 1c warrented (Fotopoulou et al., 2012). In ovarian cancer, term delivery rate following fertility saving treatment is about 30\%-\%66 and successful pregnancies are reported after adjuvant chemotherapy (Fotopoulou et al., 2012). A recurrence rate of $12 \%$ reported in patients who had fertility saving treatment and $4 \%$ of these patients died of this disease (Maltaris et al., 2007). Review of the available clinical data of recurrent disease, recurrence in a retained ovary has a significantly better prognosis compared to non-local recurrence (Satoh et al., 2010; Kajiyama et al., 2011; Kajiyama et al., 2014; Jansaka et al., 2014). Conservative treatment is not recommended in clear cell, carcinosarcoma, grade 3 and extra-ovarian disease. In stage 1 ovarian cancer, clinician should consider benefit loss ratio and determine the treatment scheme carefully. It is reasonable not to recommend adjuvant treatment for stage 1a-1b grade 1-2 patients with complete surgical staging. Although its superiority to single agent treatment is not shown in studies, most of the clinicians prefer platin based combination chemotherapy for stage 1 grade 3 and stage 1c patients (Maltaris et al., 2007).

\section{Clear Cell Histology as a Special Histologic Subtype}

In general, clear cell tumors are not graded histologically; they are accepted as being high grade when treatment sheme is planned and require adjuvant treatment. In contrast, some recent studies report low sensitivity of advanced stage clear cell carcinomas to chemotherapy, while it is suggested that recurrence rates and prognosis of early stage clear cell carcinomas are similar to that of serous carcinomas (Takada et al., 2012). Although clear cell histology was not predictive for recurrence in other studies, it was reported as being bad prognostic factor for adjuvant chemotherapy sensitivity and survival after recurrence (Kolomainen et al., 2003; Kajiyama et al., 2011). Since prognosis of clear cell ovarian cancer is comparatively poor, studies focused on alternative treatments and new therapeutic agents. These agents include temsirolismus combined with carboplatin and paclitaxel (first-line treatment in GOG 268 study) and sunitinib (second-line theraphy in GOG 254 study).

\section{Is Adjuvant Chemotherapy a Viable Option for Early Stage Unstaged Patients?}

Early stage ovarian cancer may be diagnosed in emergent surgery (eg. tortion, cyst rupture) or surgery performed for possible benign pathology (eg. ovarian cystectomy). Complete surgical staging is not performed in most of these patients. In these patients, surgical staging rather than adjuvant chemotherapy is recommended based on few but consistent available data (Trimbos et al., 2010; Dizon et al., 2008). Adjuvant chemotherapy may be recommended for patients rejecting surgical staging. There are few situations in which complete surgical staging is not necessary: borderline carcinomas with incomplete staging and grade 1 mucinous tumors (Kleppe et al., 2011; Guvenal et al., 2013).

\section{Where Should Stage 2 Disease be Evaluated?}

Most centers prefer surgical staging followed by platin based combination chemotherapy for the treatment of stage 2 ovarian cancer. Complete surgical staging is very important in determining treatment scheme. Combined analysis of GOG 95 and GOG 157 studies showed fairly high recurrence rates of stage 2 patients (Bell et al., 2006; Young et al., 2003). At present, GOG puts stage 2 patients in advanced disease group in their studies.

Chemotherapy is not recommended in stage 1 and 2 borderline ovarian tumors independent of histology and microinvasion (Patrono et al., 2013).

\section{Discussion}

Therewithal early stage disease comprises a small portion of the epithelial ovarian cancer, it has the best chance of cure. It can be problematic optimizing treatment of early stage ovarian cancers due to the lack of randomized studies and the variety of controversial issues. The value of targeted agents which are increasingly utilized in advanced-stage disease is unknown. Careful consideration of stage of the disease, histologic subtypes, degree of surgical radicality, potential desire of fertility preservation and rational recurrence risk must be warranted while planning the adjuvant treatment and well designed prospective studies are needed to determine the optimal chemotherapy regimen for these patients. 


\section{References}

Adams G, Zekri J, Wong H, Walking J, Green JA (2010). Platinumbased adjuvant chemotherapy for early-stage epithelial ovarian cancer: single or combination chemotherapy? BJOG, 117, 1459-67.

Arikan SK, Kasap B, Yetimalar H et al (2014). Impact of prognostic factors on survival rates in patients with ovarian carcinoma. Asian Pac J Cancer Prev, 15, 6087-94.

Bell J, Brady MF, Young RC et al (2006). Randomized phase III trial of three versus six cycles. Gynecol Oncol, 102, 432-9.

Chan JK, Tian C, Teoh D et al, (2010). Survival after recurrence in early-stage high-risk epithelial ovarian cancer: a Gynecologic Oncology Group study. Gynecol Oncol, 116, 307-11.

Chan JK, Tian C, Fleming GF, et al (2010). The potential benefit of 6 vs. 3 cycles of chemotherapy in subsets of women with early-stage high-risk epithelial ovarian cancer: an exploratory analysis of a gynecologic oncology group study. Gynecol Oncol, 116, 301.

Colombo N, Guthrie D, Chiari S et al (2003). International Collaborative Ovarian Neoplasm trial 1: a randomized trial of adjuvant chemotherapy in women with early-stage ovarian cancer. J Natl Cancer Inst, 95, 125-32.

Collinson F, Qian W, Fossati R, et al; ICON1 collaborators, (2014). Optimal treatment of early-stage ovarian cancer. Ann Oncol, 25, 1165-71.

Dizon DS, Restivo A, Lomme M, et al (2008). For women receiving chemotherapy for clinically apparent early ovarian cancer, is there a benefit to surgical staging? Am J Clin Oncol, 31, 39.

Fotopoulou C, Braicu I, Sehouli J (2012). Fertility-sparing surgery in early epithelial ovarian cancer: a viable option? Obstet Gynecol Int, 2012, 238061.

Guvenal T, Dursun P, Hasdemir P, et al (2013). Effect of surgical staging on 539 patients with borderline ovarian tumors: a turkish gynecologic oncology group study. Gynecologic Oncology, 131, 546-50.

Jansaka N, Suprasert P (2014). Survival outcomes of recurrent epithelial ovarian cancer: experience from a Thailand northern tertiary care center. Asian Pac J Cancer Prev, 15, $10837-40$.

Kajiyama H, Shibata K, Mizuno M, et al (2011). Fertility-sparing surgery in young women with mucinous adenocarcinoma of the ovary. Gynecol Oncol, 122, 334-8.

Kajiyama H, Shibata K, Mizuno M, et al (2011). Fertility-sparing surgery in patients with clear-cell carcinoma of the ovary: is it possible? Hum Reprod, 26, 3297-02.

Kajiyama H (2014). Fertility sparing surgery in patients with early stage epithelial ovarian cancer: implication of survival analysis and lymphadenectomy. J Gynecol Oncol, 25, 270-1.

Karimi Zarchi M, Mousavi A, Gilani MM, et al (2011). Fertility sparing treatments in young patients with gynecological cancers: Iranian experience and literature review. Asian Pac J Cancer Prev, 12, 1887-92.

Kleppe M, Wang T, Van Gorp T, et al (2011). Lymph node metastasis in stages I and II ovarian cancer: a review. Gynecol Oncol, 123, 610-4.

Kolomainen DF, A'Hern R, Coxon FY, et al (2003). Can patients with relapsed, previously untreated, stage I epithelial ovarian cancer be successfully treated with salvage therapy? J Clin Oncol, 21, 3113-8.

Lazarov N, Lazarov L, Lazarov S (2013). The role of adjuvant chemotherapy as a prognostic factor in patients with early stage of epithelian ovarian cancer (I - II stage). Akush Ginekol (Sofiia), 52, 58-61.

Malpica A, Deavers MT, Lu K, et al (2004). Grading ovarian serous carcinoma using a two-tier system. Am J Surg Pathol, 28, 496-504.

Maltaris T, Seufert R, Fischl F (2007). The effect of cancer treatment on female fertility and strategies for preserving fertility. Eur J Obstet Gynecol Reprod Biol, 130, 148-55.

Mannel RS, Brady MF, Kohn EC, et al (2011). A randomized phase III trial of IV carboplatin and paclitaxel $\times 3$ courses followed by observation versus weekly maintenance low-dose paclitaxel in patients with early-stage ovarian carcinoma: a Gynecologic Oncology Group Study. Gynecol Oncol, 122, 89-94.

National Comprehensive Cancer Network (NCCN), (2014). http://www.nccn.org/.

NCT01462890. http://www.ago-ovar.de/en/trials-closedtrials-15.html.

Patrono MG, Minig L, Diaz-Padilla I, et al (2013). Borderline tumours of the ovary, current controversies regarding their. Ecancermedicalscience, 7, 379.

Perren TJ, Swart AM, Pfisterer J et al; ICON7 Investigators (2011). A phase 3 trial of bevacizumab in ovarian cancer, 365, 2484-96.

Raja FA, Chopra N, Ledermann JA (2012). Optimal first-line treatment in ovarian cancer. Ann Oncol, 23, 118-127.

Satoh T, Hatae M, Watanabe Y, et al (2010). Outcomes of fertility-sparing surgery for stage I epithelial ovarian cancer: a proposal for patient selection. J Clin Oncol, 28, 1727-32.

Siegel R, Naishadham D, Jemal A (2013). Cancer statistics. CA Cancer J Clin, 63, 11-30.

Takada T, Iwase H, Iitsuka C, et al (2012). Adjuvant chemotherapy for stage I clear cell carcinoma of the ovary: an analysis of fully staged patients. Int J Gynecol Cancer, 22, 573-8.

Trimbos JB, Vergote I, Bolis G et al (2003). Impact of adjuvant chemotherapy and surgical staging in early-stage ovarian carcinoma: european organisation for research and treatment of cancer-adjuvant chemotherapy in ovarian neoplasm trial. $J$ Natl, 95, 113-25.

Trimbos B, Timmers P, Pecorelli S et al (2010). Surgical staging and treatment of early ovarian cancer: long-term analysis from a randomized trial. J Natl Cancer Inst, 102, 982-7.

Tognon G, Carnazza M, Ragnoli M et al (2013). Prognostic factors in early-stage ovarian cancer. Ecancermedicalscience, 7, 325 .

Winter-Roach BA, Kitchener HC, Lawrie TA (2012). Adjuvant (post-surgery) chemotherapy for early stage epithelial ovarian cancer. Cochrane Database Syst Rev, 3, CD004706.

Young RC, Brady MF, Nieberg RK, et al (2003). Adjuvant treatment for early ovarian cancer: a randomized phase III trial of intraperitoneal 32P or intravenous cyclophosphamide and cisplatin--a gynecologic oncology group study. J Clin Oncol, 21, 4350. 